$16^{\text {th }}$ International Conference on

AEROSPACE SCIENCES \& AVIATION TECHNOLOGY,

ASAT - 16 - May 26 - 28, 2015, E-Mail: asat@ mtc.edu.eg

Military Technical College, Kobry Elkobbah, Cairo, Egypt

Tel : +(202) 24025292 - 24036138, Fax: +(202) 22621908

\title{
A Comparative Analysis of CFO Estimation Techniques in OFDM Systems
}

\author{
Shaimaa ElSayed ${ }^{*}$, K.A. ElBarbary ${ }^{\dagger}$ and Ragab M. El-Sagheer ${ }^{\S}$
}

\begin{abstract}
OFDM systems are sensitive to Carrier Frequency Offsets (CFO) that results in performance's degradation. Researchers have proposed various CFO compensation techniques. Some estimates the CFO's value then compensates for it using preamble methods. Others estimate the CFO value using the inherent construction of the time-domain OFDM symbol. Our proposed technique changes the conventional structure of the OFDM symbol to allow estimating the CFO values over a wide range. CFO estimation range, accuracy, transmitted data-rate, system complexity, and the compatibility of a specific estimator to be used in different applications are the most important parameters to be considered in researches. In this paper, a comparative analysis between preamble techniques (Moose, Training Symbol) and the OFDM internal construction-based estimators including blind and proposed schemes will be discussed. Simulations show the trade-offs between those techniques.
\end{abstract}

Keywords: OFDM; carrier frequency offset; convolution; preamble.

\section{Introduction}

OFDM systems are more sensitive to carrier frequency offsets (CFO) than single carrier systems. In general, Frequency offset is defined as the difference between the nominal frequency and the actual carrier frequency. In OFDM, the variation between those frequencies gives rise to a shift in the frequency domain. This in turn could lead to degradation in the system performance [1]. The motivation behind researches is therefore to overcome the effect of this frequency drift. In general, researches were classified into two groups. The first group includes the CFO sensitivity reduction techniques that aim to reduce the OFDM sensitivity against the CFO. These include Self Cancellation (SC) [2] and Conjugate Cancellation (CC) schemes [3]. Those methods can mitigate the effect of the CFO for small values that not exceed half the subcarrier spacing at the price of sever reduction in the transmitted data rate by a half value at least. The second group includes the CFO estimation and correction techniques [4], [5] in which the CFO is estimated and corrected at the receiver side. In general, this group can be classified into two categories. First, non-data aided category in which blind estimation of CFO value is determined [5]. Blind estimation algorithms [6] use exclusively the statistic properties of transmitted signal by the help of the cyclic prefix (CP) for estimation purposes. Unfortunately, CP-based estimators suffer from low CFO estimation range that is $\varepsilon \leq 0.5$, where $(\varepsilon)$ is defined as a ratio of the value of CFO to the subcarrier spacing.

\footnotetext{
* Lecturer Assistant, Dept. of EECT, Modern Academy, Ma'adi, Egypt, eng sh3e@yahoo.com

${ }^{\dagger}$ Professor and Head of Electric Engineering Department, Suez Canal University, Egypt

${ }^{\S}$ Lecturer, Dept. of Electrical Communication, Al-Azhar University, Cairo, Egypt.
} 
Second category is the data aided category in which the CFO can be estimated by the help of a preamble inserted at the begging of a frame. Moose in [7], which can estimate CFO values over $\varepsilon \leq 0.5$, and Training Symbol method in [8], that able to extend this estimation range, are examples of these preamble methods. Although preamble methods have the advantages of giving high accuracy performance with a minor reduction in transmitted data rate as a result of transmitting repetitive sequence, they have some drawbacks. One drawback of those algorithms is their high computational complexity which is expressed in terms of complex multiplications and additions. Unlike blind estimation method in [5], another drawback for the preamble methods, in general, is its sacral ability to update the CFO estimated value during the frame. This leads to excessive errors which can be enhanced in blind estimators' methods.

In our proposed scheme, the CFO value can be estimated over a wide range, as in preamble methods in [8], and also can be updated many times through the frame, as in CP-based estimators in [5], [6]. The competition between all of these techniques is to estimate higher CFO values with lower possible reduction in transmitted data rates keeping high estimation accuracy with low complexity. A comparative analysis between different CFO estimation methods are discussed in this paper showing the tradeoffs between them. The comparisons are made between preamble methods of Moose, training symbol and CP-based (blind estimators), our proposed estimator.

The rest of the paper is organized as follows: In section 2, OFDM system description has been described. The effect of CFO in the system has been discussed in section 3 . In section 4, Moose, cyclic prefix, training symbol and our proposed estimation techniques have been described. A trade-off comparison between those different schemes is discussed in section 5 . In section 6, simulation results have been analyzed. Finally, conclusions of the comparisons are shown in section 7 showing the tradeoffs between them.

\section{OFDM System Basic Structure}

This section explains the basic OFDM implementation in a CFO free channel. In OFDM system, the binary serial data is mapped into M-PSK or M-QAM modulator forming $\{X(k)\}, k=0: N-1$ before transforming into the signal block $\{x(n)\}, n=0: N-1$ by IFFT which given by:

$$
x(n)=\frac{1}{N} \sum_{k=0}^{N-1} X(k) e^{\frac{j 2 \pi n k}{N}}, n=0: N-1
$$

Where, $\mathrm{k}$ is the subcarrier index at the transmitter side and each frequency $(2 \pi \mathrm{k} / \mathrm{N})$ represents a carrier. After parallel-to-serial conversion $(\mathrm{P} / \mathrm{S})$, a cyclic prefix $(\mathrm{CP})$ is added with length $\mathrm{N}_{\mathrm{g}}$ that is larger than the delay in the multipath channel to prevent intersymbol interference (ISI). This will lead to a reduction in the transmitted data rate. However, the time-domain transmitted signal is then $\{\mathrm{s}(\mathrm{n})\}, \mathrm{n}=-\mathrm{N}_{\mathrm{g}}: \mathrm{N}-1$. If this signal was propagated in Additive White Gaussian Noise (AWGN) channel, the received samples $\{r(n)\}, n=-N_{g}: N-1$ will have the same length as $\{s(n)\}$. But if the channel was a multipath channel with number of taps equals to $\mathrm{v}$, then the received signal $\{\mathrm{r}(\mathrm{n})\}, \mathrm{n}=$ $\left(-\mathrm{N}_{\mathrm{g}}: \mathrm{N}+\mathrm{v}-2\right)$ will has a length of $\left(\mathrm{N}+\mathrm{N}_{\mathrm{g}}+\mathrm{v}-1\right)$ this is due to the linear convolution process between the transmitted signal and the multipath channel [9]. At the receiver side and after the serial-to-parallel ( $\mathrm{S} / \mathrm{P})$ conversion, the cyclic prefix is removed as it contains no information and samples at the tail of indices $n=\{N$ : end $\}$ which are due to linear convolution are neglected. The obtained received data in the time domain $\{y(n)\}, n=0: N-$ 
1 are then converted to the frequency domain $\{\mathrm{Y}(\mathrm{l})\}, \mathrm{n}=0: \mathrm{N}-1$ using the fast Fourier transform (FFT) algorithm. An equalization process is needed in case of multipath channel to cancel the effects of its components. The output is then demodulated to obtain the estimated binary data sequence. OFDM general block diagram is shown in Fig.1.

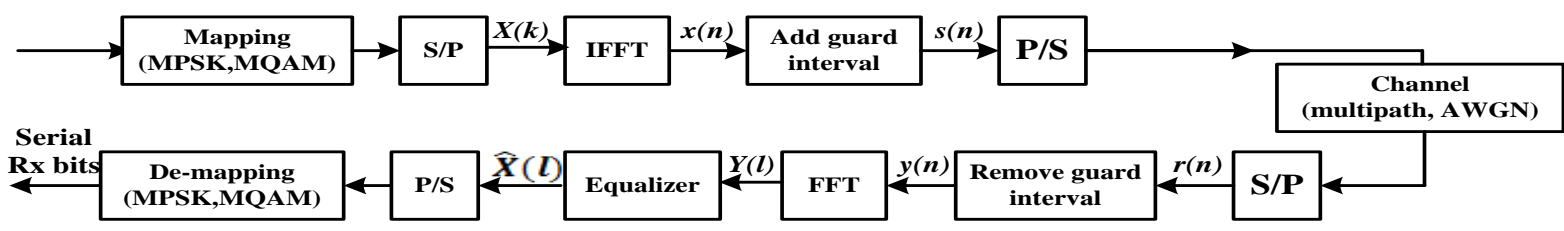

Fig. 1. OFDM general block diagram

\section{Carrier Frequency Offset in OFDM}

The use of OFDM systems with a high number of subcarriers has some drawbacks. The major drawback is its high sensitivity to synchronization non idealities between the transmitter and receiver oscillators as well as the Doppler's effect [4]. These non idealities result in a Carrier frequency offset (CFO) which causes degradation in the system performance. This is because the CFO results in an amplitude reduction of the useful signal and provokes interference between adjacent subcarriers that is named inter-carrier interference (ICI). For AWGN channel, the received time-domain signal $y(n)$, ignoring CP part, is given by:

$y(n)=x(n) e^{\frac{j 2 \pi \varepsilon n}{N}}+w(n)$

Where $\varepsilon$, is the normalized CFO which is the value of carrier frequency offset $\Delta f$ normalized by the carrier frequency spacing $f_{\text {sub }}$ and $w(n)$ is a zero-mean (AWGN) noise. Since the shift affects the frequency components, the CFO is modeled as a complex multiplicative distortion of the received data in the time domain. This is shown in Eq. (2) which assumes that all subcarriers experience the same shift $\varepsilon$. The received signal in frequency domain after fast Fourier transform block, at $l^{\text {th }}$ subcarrier can be written as:

$Y(l)=X(l) S(0)+\sum_{\substack{k=0 \\ k \neq l}}^{N-1} X(k) S(k-l)+W(l)$

Where, $l$ is the subcarrier index at the receiver side, $\{S(k-l)\}$ are the complex coefficients of the ICI components in the received signal and $W(l)$ is the FFT of $w(n)$. the complex coefficients $\{S(k-l)\}$ are given by [2]:

$S(k-l)=e^{j \pi[k+\varepsilon-l]\left(1-\frac{1}{N}\right)} * \frac{\sin \pi(k+\varepsilon-l)}{N \sin \left(\frac{\pi}{N}(k+\varepsilon-l)\right)}$

In a multipath channel, Eq. (3) will be:

$Y(l)=X(l) H(l) S(0)+\left(\sum_{\substack{k=0 \\ k \neq 1}}^{N-1} X(k) H(l) S(k-l)+W(l)\right)$

Where $H(l)$ is FFT of the channel impulse response $h(n)$. In general, Equations (3-5) imply that, the CFO causes loss of subcarrier orthogonality and thus lead to ICI, which severely degrades the system performance. So before demodulation, CFO estimation and compensation is of great importance in OFDM communications. If the value of frequency 
offset is estimated at the receiver, the ICI distortion of data symbols can be reduced by using a process that is opposite to Eq. (2) as:

$\hat{x}(n)=\{x(n)+w(n)\} e^{\frac{-j 2 \pi \varepsilon n}{N}}$

So at high signal to noise ratios and using a high accuracy estimator method, $\hat{x}(n)=x(n)$.

\section{CFO Estimation Techniques}

This section analyzes different CFO estimation methods that depend on transmitting repeated samples in time domain. This repeated samples can either be preamble and sent at the start of the frame as used in Moose and training symbol schemes or be a part of the OFDM symbol structure and contained in every OFDM block symbol as used in cyclic prefix and proposed estimation methods. In all cases, the structure of this repeating data is known at receiver side but its values are unknown.

\subsection{Moose Technique}

In [7], Moose proposed a data aided scheme using a preamble in the beginning of the frame that is two OFDM blocks length. Another name of Moose technique is data-driven technique [10]. The basis here is that, the same data contained in the whole OFDM block symbol is repeated and the phase value of the same sub-carrier between consecutive symbols is compared for all subcarriers. The offset is determined by the maximum likelihood estimation algorithm (MLE). For such a frame construction, a two received time domain OFDM symbols which have the same data and corrupted by CFO in a multipath channel is given by:

$$
\begin{aligned}
& y(n)=\frac{1}{N} \sum_{k=0}^{N-1} X(k) H(k) e^{\frac{j 2 \pi n(k+\varepsilon)}{N}}+w(n) \quad, n=0: 2 N-1 \\
& , Y_{1}(l)=\sum_{n=0}^{N-1} y(n) e^{-\frac{j 2 \pi n l}{N}}+W_{1}(l) \\
& , Y_{2}(l)=\sum_{n=N}^{2 N-1} y(n) e^{-\frac{j 2 \pi n l}{N}}+W_{2}(l)=\sum_{n=0}^{N-1} y(n+N) e^{-\frac{j 2 \pi n l}{N}}+W_{2}(l), l=0: N-1
\end{aligned}
$$

Where $Y_{1}(l)$ and $Y_{2}(l)$ are the $l^{\text {th }}$ element of the first and second $N$-points samples of Equation (7) respectively. So, considering no CP part, at high SNR values:

$$
\begin{aligned}
& y(n+N)=y(n) e^{\frac{j 2 \pi \varepsilon N}{N}}=y(n) e^{j 2 \pi \varepsilon} \\
& \therefore \quad Y_{2}(l)=Y_{1}(l) e^{j 2 \pi \varepsilon}
\end{aligned}
$$

In Moose's technique, the CFO is estimated in frequency domain using Eq. (10). The maximum likelihood estimate (MLE) of $\varepsilon$ is given by [7]:

$$
\hat{\varepsilon}_{\text {Moose }}=\frac{1}{2 \pi} * \arg \left\{\sum_{n=0}^{N-1} Y_{2}(l) Y_{1}^{*}(l)\right\}
$$

Since the range of $\arg \{$.$\} operation [11] is limited by (-\pi:+\pi)$, the CFO estimation range in Moose's technique is limited by $[(-\pi:+\pi) / 2 \pi]=[-0.5:+0.5]$. But if we took the CP-part into considerations, Eq. (10) would be: 


$$
\begin{aligned}
& y_{n+N}=y_{n} e^{\frac{j 2 \pi \varepsilon\left(N+N_{g}\right)}{N}} \\
& \therefore Y_{2}(l)=Y_{1}(l) e^{\frac{j 2 \pi \varepsilon\left(N+N_{g}\right)}{N}} \\
& \therefore \hat{\varepsilon}_{\text {Moose }}=\frac{N}{2 \pi\left(N+N_{g}\right)} * \arg \left\{\sum_{n=0}^{N-1} Y_{2}(l) Y_{1}^{*}(l)\right\}
\end{aligned}
$$

Hence, the estimation range will be limited by $\left[(-\pi:+\pi) \mathrm{N} / 2 \pi\left(N+N_{g}\right)\right]=\left[\left(-N / 2\left(N+N_{g}\right)\right):\left(+N / 2\left(N+N_{g}\right)\right)\right]$ which is reduced than $[-0.5:+0.5]$. For example if $\left(N_{g}=N / 4\right)$, the estimation range in Moose's technique will be within $[-0.4:+0.4]$ range. So, this scheme suffers from lowering CFO estimation range as $\left(N_{g} / N\right)$ increases. Also a reduction in transmitted bandwidth efficiency occurred as the requirement of repeating the whole samples existed in the OFDM symbol. Taking the redundancy in the $N_{g}$ part into considerations, if the repetition is used once at the beginning of the frame which contains $\mathcal{M}$-OFDM block symbols, then the number of transmitted mapped data will be reduced to $\left[\{(N / 2)+(\mathcal{M}-2) N\} /\left(N+N_{g}\right)\right]$. This technique takes the advantage of high accuracy estimation as the averaging process takes place over the whole samples contained in the OFDM block. The computational complexity in this technique, according to Equations (11) and (13), needs N multiplications. So, it increases with increasing the used number of orthogonal subcarriers.

\subsection{CP-Based Technique}

This technique estimates the CFO by the help of the redundancy contained in CP. Considering the correlation between the samples in CP and their copies which are spaced $N$ samples apart, then:

$E\left\{r(n) r^{*}(n+N)\right\}=\sigma_{s}^{2} e^{j 2 \pi \varepsilon}, \quad n=-N_{g}:-1$

The $E\{\cdot\}$ expresses here the average or expected value and $\sigma_{s}^{2}$ is the power of the sample. So,

$\hat{\varepsilon}_{C P}=\frac{1}{2 \pi} * \arg \left\{\sum_{n=-N_{g}}^{-1} r_{p}^{*}[n] r_{p}[n+N]\right\}$

Where $p$ and $r[\cdot]$ indicate an OFDM symbol index number and the OFDM received symbol before removing $\mathrm{CP}$, respectively. Equation (15) is derived from the log-likelihood function $\Lambda(\varepsilon)$ that is discussed and analyzed in [6], [12]. Maximum Likelihood (ML) estimation of $\varepsilon$ is the argument that maximizes $\Lambda(\varepsilon)$ then yields Eq. (15). As the CFO estimation range in C.P. scheme is limited by the $\arg \{$.$\} operation, it then limited to be$ within $[(-\pi:+\pi) / 2 \pi]=[-0.5:+0.5]$. Unlike Moose's scheme, CP length does not affect this estimation range. To increase the accuracy in a noisy system, P-OFDM symbols are used in the estimation process [5], [13] which expressed as:

$$
\hat{\varepsilon}_{C P}=\frac{1}{2 \pi} * \arg \left\{\sum_{p=0}^{P-1} \sum_{n=-N_{g}}^{-1} r_{p}^{*}[n] r_{p}[n+N]\right\}
$$

In multipath channels, the received signal is received from $v$-paths. To avoid inter-symbol interference (ISI), the samples in CP portion should be at least as long as channel order $v$. Another advantage of using CP for the guard symbol is that it helps to maintain the receiver carrier synchronization; the utilization of $\mathrm{CP}$ enables OFDM system to convert a frequency selective channel into a parallel collection of frequency flat channels, leading to greatly 
simplified equalizer design [12]. However, in the multipath channel, a part of the $\mathrm{CP}$ is corrupted by the preceding symbols. So, the CFO estimation process needs to be within the uncorrupted part as:

$\hat{\varepsilon}_{C P}=\frac{1}{2 \pi} * \arg \left\{\sum_{p=0}^{P-1} \sum_{n=-N_{g}+v-1}^{-1} r_{p}^{*}[n] r_{p}[n+N]\right\}$

Where $r(n)=\grave{x}(n) e^{\frac{j 2 \pi \varepsilon n}{N}}+w(n)$, and $\grave{x}(n)=x(n) \star h(n)$ and $\star \quad$ indicates linear convolution process, $h(n)$ is the channel impulse response with length $(v)$ and $\grave{x}(n)$ is the received OFDM symbol with length $\left(N+N_{g}+v-1\right)$. Because the number of samples in the CP affects the performance of the estimator) [6], more OFDM symbols will be needed than that used in AWGN channel to enhance the accuracy in multipath channels with longer delays according to Eq. (17). The optimized transmitted bandwidth efficiency can be taken for OFDM transmission in AWGN channel; as there is no need for using CP in this channel. Using cyclic prefix, in a multipath channel results in a reduction of transmitted bandwidth efficiency by a factor of $\left[N /\left(N+N_{g}\right)\right]$. The computational complexity here is determined by $\mathrm{N}_{\mathrm{g}}$ multiplications $\mathrm{P}$ times.

\subsection{Training Symbol Technique}

We have seen that, the CFO estimation technique using $\mathrm{CP}$ can estimate the $\mathrm{CFO}$ only within the range $[-0.5:+0.5]$. Since the normalized CFO can be larger than this value [14] especially if the offset was due to the mismatch between the local oscillators; another technique that can estimate within a wider range may be preferred.

The normalized $\mathrm{CFO}$ estimation range can be increased by reducing the distance between two blocks of samples for correlation [8]. A preamble technique that uses the Training Symbol (TS) concept was proposed in [7], [15]. In this technique, a transmitter sends a preamble in the beginning of a frame with the same length of one OFDM symbol block. This preamble contains training symbols with $D$ repetitive patterns or sub-blocks in the time domain, which can be generated by taking the IFFT of a comb-type signal in the frequency domain given as:

$$
X(k)=\left\{\begin{array}{lr}
A_{m} \text { if } k=D \cdot i, & i=0:(N / D)-1 \\
0 & \text { otherwise }
\end{array}\right.
$$

Where $A_{m}$ represents an $\mathrm{M}$-ary symbol and $(N / D)$ is an integer number. The normalized carrier frequency offset can be estimated by the correlation of the repeated identical parts as:

$$
\hat{\varepsilon}_{T S}=\frac{D}{2 \pi} * \arg \left\{\sum_{n=0}^{(N / D)-1} y_{p}^{*}[n] y_{p}[n+N / D]\right\}
$$

Here, $y[\cdot]$ is the received sample after removing the cyclic prefix part. In this technique, the value of $D$ determines the estimation range which is limited by $[D *(-\pi:+\pi) / 2 \pi]=$ $[-D / 2:+D / 2]$. So, the range of $\left|\hat{\varepsilon}_{T S}\right| \leq(D / 2)$ becomes wider as $D$ increases at the price of reducing the estimation accuracy. This means, in TS scheme, the estimation accuracy is reduced as the number of samples contained in the preamble and used for the computation of correlation in time domain is reduced by the factor $(1 / D)$ [8]. Using average method, this estimator exploits the correlations of the samples from the receiver filter [15]:

$A(m)=\frac{1}{N-m B} \sum_{n=m B}^{N-1} y_{p}[n] y_{p}{ }^{*}[n-m B], m=0: \beta$

Where $\beta$ is a design parameter which is less than or equals to $(D-1)$. By computing the angles $\{\varphi(m)\}$, the normalized CFO can be estimated, where: 
$\varphi(m)=[\arg \{A(m)\}-\arg \{A(m-1)\}]_{2 \pi}, m=1: \beta$

Here, $[u]_{2 \pi}$ denotes modulo- $2 \pi$ operation (it reduces $u$ to the interval $[-\pi: \pi]$. The best linear unbiased estimator of this method can be expressed as [15]:

$\hat{\varepsilon}_{T S}=\frac{D}{2 \pi} \sum_{\mathrm{m}=1}^{\beta} \mathrm{w}(\mathrm{m}) \emptyset(\mathrm{m})$

The weighting function $w(m)$ is given by:

$w(m)=\frac{3(D-m)(D-m+1)-\beta(D-\beta)}{\beta\left(4 \beta^{2}-6 D \beta+3 D^{2}-1\right)}$

At high signal to noise ratios, the normalized $\mathrm{CFO}$ can be found to be limited by the interval $\left|\hat{\varepsilon}_{T S}\right| \leq(D / 2)$. Although using average method increases the scheme's accuracy without reducing the estimation range of $\mathrm{CFO}$, but this is done at the price of increasing the computational complexity. The computational complexity increases in this technique as it fully exploit all the possible $[D(D-1) / 2]$ sets of repetition patterns for the auto-correlation to increase its accuracy. Each correlation function $A(m)$ then needs $\{[N(1-m / D)], m=$ $0: D-1\}$ multiplications at $\beta=D-1$. As this redundancy is added once at the front of each frame, the transmitted bandwidth is best to be determined in normalized form. Consider a frame contains $\mathcal{M}$-OFDM symbol blocks, the transmitted bandwidth in AWGN channel can be computed as $[(\mathcal{M}-1) / \mathcal{M}]$. While in a multipath channel and as a result of adding a cyclic prefix part, the number of transmitted mapped data within the frame with length $\mathcal{M}$ will be limited to $\left[\{(\mathcal{M}-1) N\} / \mathcal{M}\left(N+N_{g}\right)\right]$.

\subsection{Proposed Technique}

In proposed technique, the CFO value can be estimated over a wide range [14] as in TS preamble technique beside the availability to be updated many times through the frame, as in CP-based estimators' techniques. In this proposed technique, the distance between two blocks of samples to be correlated is reduced using a new OFDM symbol structure. In this paper, we will name our proposed technique by "Training Prefix (T.P.)" technique.

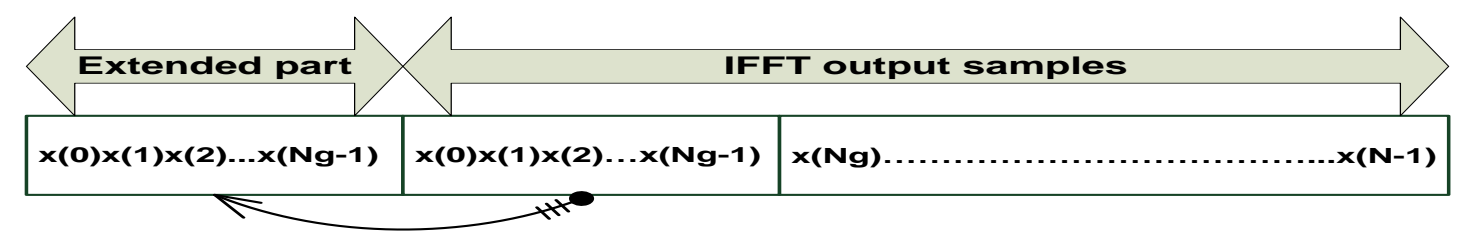

Fig. 2. OFDM symbol structure used in TP technique for AWGN channel

Considering only AWGN channel, this OFDM symbol structure looks like the one used in CP technique and shown in Fig. 2. But instead of prefixing the OFDM symbol at the start by the last $N_{g}$ samples of the OFDM symbol, it is extended by the first $N_{g}$ samples of the same symbol, taking the form:

$s(n)= \begin{cases}x\left(n+N_{g}\right) & \text { if } \quad-N_{g} \leq n<0 \\ x(n) & \text { if } \quad 0 \leq n \leq N-1\end{cases}$

Here, the CFO can be estimated from the product of extended samples and their copy that spaced $N_{g}$ samples apart as:

$\hat{\varepsilon}_{T P}=\left(\frac{N}{2 \pi N_{g}}\right) \arg \left\{r^{*}[n] r\left[n+N_{g}\right]\right\}, n=-1,-2, \ldots,-N_{g}$ 
Therefore, the phase difference between correlated samples caused by CFO is $\left(2 \pi N_{g} \varepsilon / N\right)$, resulting in extending the estimation range to be $\varepsilon=\left[(-\pi:+\pi) /\left(2 \pi N_{g} / N\right)\right]=\left[(-N:+N) / 2 N_{g}\right]$ which depends on $\left(N_{g} / N\right)$ ratio. As in $\mathrm{CP}$-scheme the average of Eq. (25) can be taken over the samples in extended interval to reduce the noise effect. The accuracy can be increased if $N$ is increased or $P$-OFDM symbols are employed as:

$$
\hat{\varepsilon}_{T P}=\left(\frac{N}{2 \pi N_{g}}\right) \arg \left\{\sum_{p=0}^{P-1} \sum_{n=-N_{g}}^{-1} r_{p}^{*}[n] r_{p}\left[n+N_{g}\right]\right\}
$$

In this new OFDM symbol structure, considering multipath transmission, the linear channel convolution could not be converted into circular convolution as in previous mentioned techniques that uses cyclic prefix. That is because the extended $N_{g}$ samples are appended after the IFFT stage i.e., resulting in discontinuity in time domain. This discontinuity affects the equalization process at receiver making it harder [14]. So, some manipulation was performed to the symbol structure for simpler equalization process. This is done by adding other extra $N_{g}$ samples that are copies of last $N_{g}$ samples of $x(n)$ and located in front of $s(n)$ as follow:

$$
s(n)=\left\{\begin{array}{clc}
x\left(n+N+N_{g}\right) & \text { if } & -2 N_{g} \leq n<-N_{g} \\
x\left(n+N_{g}\right) & \text { if } & -N_{g} \leq n<0 \\
x(n) & \text { if } & 0 \leq n \leq N-1
\end{array}\right.
$$

Besides permitting simple equalization with same complexity as in conventional OFDM system, the first added $N_{g}$ samples acts as a guard interval in multipath channels. Although this symbol structure makes the equalization process be simple, it causes a minor reduction in transmitted bandwidth efficiency by a factor of $\left[N /\left(N+2 N_{g}\right)\right]$. In general, the data rate got at $\left(N_{g} / 2 N\right)$ in standard OFDM system is equivalent to that at $\left(N_{g} / N\right)$ in case of the proposed scheme. This is due to the redundancy of added extra $2 N_{g}$ samples. However, in T.P. proposed technique, the received OFDM symbol from the multipath channel is the result of linear convolution of OFDM symbol of length $\left[N+2 N_{g}\right]$ with the channel impulse response of length $v$. The equivalent received OFDM symbol can be written in a mathematical form as:

$r(n)=\grave{s}(n) e^{\frac{j 2 \pi \varepsilon n}{N}},-2 N_{g} \leq n \leq N+v-2$

The CFO estimated value depends on the relation between the analogous received samples that spaced $N_{g}$ samples apart. In a multipath channel, the number of these analogous samples is decreased as the channel length increase. To reduce the noise effect, the CFO can then be estimated by averaging the product of the analogous received samples over P-OFDM symbols as:

$\hat{\varepsilon}_{T P}=\left(\frac{N}{2 \pi N_{g}}\right) \arg \left\{\sum_{p=0}^{P-1} \sum_{n=-N_{g}+v-1}^{-1} r_{p}{ }^{*}[n] r_{p}\left[n+N_{g}\right]\right\}$

It is obvious that, the number of samples in $N_{g}$ participates in determining the estimation accuracy. The effect of $\mathrm{CFO}$ on received signal can then be reduced by multiplying the received OFDM symbol by the conjugation of $e^{j 2 \pi \varepsilon n / N}$, resulting in $r_{c}(n),-N_{g} \leq n \leq N-$ 1. The CFO estimation and correction stage is declared in Fig. 3. As shown, after the correction stage, a replacement of $r_{c}(n), 0 \leq n \leq N_{g}-1$ by $r_{c}(n),-N_{g} \leq n \leq-1$ takes 
place. Now, the first $N_{g}$ samples can be removed to get $y(n)$ with the same size of FFT. By this way, the linear channel convolution is converted into circular convolution which makes the equalization process simple using one-tap frequency domain equalizer as used in $\mathrm{CP}$ scheme case. As shown, this is done without needing any extra design complexity.

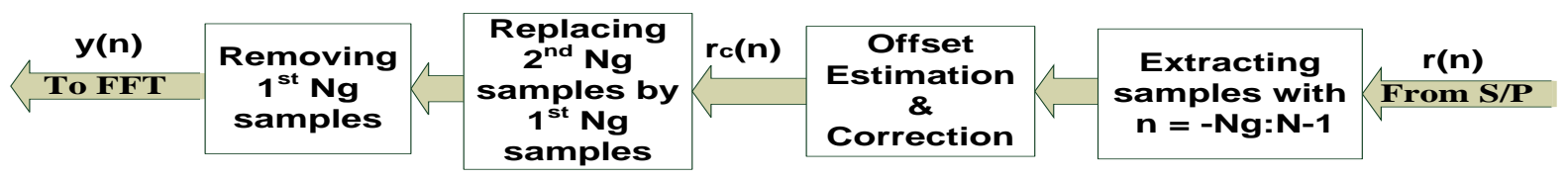

Fig. 3. Offset estimation and correction stage of TP scheme for multipath channel

The difference between linear and circular convolution can be found in [14]. Unlike preamble estimators, our proposed estimator is able to update the CFO estimated value many times during the frame; as it adds the redundancy at every OFDM symbol. Beside this, the proposed OFDM symbol structure enables simple equalization process with the advantage of increasing the CFO estimation range. This is because of the redundancy contained in each transmitted OFDM symbol block that enables the estimation to be updated every block. As blind estimator scheme, the computational complexity here is determined by $N_{g}$ multiplications $\mathrm{P}$ times.

\section{A Comparative Trade-offs Discussion}

The transmitted bandwidth efficiency, estimation accuracy, CFO estimation range and complexity are considered as the most important parameters in choosing the suitable estimation technique. Also the ability to update the estimated value during a frame plays an important role to choose it. This section demonstrates comparative tradeoffs between the predescribed four schemes.

\subsection{Transmission Bandwidth Efficiency}

In a multipath channel, there is a need of transmitting a guard interval in the front of the OFDM signal that can be filled with a repeated data samples leading to a reduction in transmitted bandwidth efficiency. This reduction differs according to used technique and depends on the number of redundancy samples added to the transmitted OFDM symbol. As $\mathrm{CP}$ part is needed to prevent ISI, it is used in all techniques. So, CP has the highest bandwidth efficiency over all other techniques. Another extra redundancy is required for Moose, TS and TP schemes which reduces their transmission efficiency than CP technique. Because Moose and TS techniques add this redundancy as a preamble, it is sent once at the beginning of the frame. Thus in case of long frame length and according to $\left(N_{g} / N\right)$ ratio, they become more bandwidth efficiency than TP scheme. This is because in proposed TP scheme the redundancy is contained in every transmitted OFDM block symbol. However, the data rate got at $\left(N_{g} / 2 N\right)$ in standard OFDM system is equivalent to that at $\left(N_{g} / N\right)$ in case of the proposed scheme. Note that, the length of cyclic prefix portion affects the transmitted bandwidth efficiency for all Moose, CP, TS and TP techniques.

\subsection{Estimation Accuracy}

The estimator accuracy increases when the number of samples used in the estimation process increase. As the Moose technique uses the whole $N$-samples contained in the OFDM block symbol, it has high estimation accuracy. Also, averaging process that takes place over two consecutive sub-blocks for all repetitive patterns increases the estimation accuracy in TS 
technique. In both $\mathrm{CP}$ and proposed $\mathrm{TP}$ techniques, the number of samples used in the estimation process is reduced to be a part of the OFDM symbol block. This reduces the estimation accuracy to be low if the length of the OFDM symbol is low. This accuracy can be increased if the averaging over $P$-OFDM symbols or longer CP samples take place. However, this can be avoided if the length of the OFDM is increased. Discontinuity in time domain of proposed OFDM symbol structure lowers the accuracy of the proposed scheme compared to the conventional CP-based estimator method. This can be difference can be ignored if we used high number of orthogonal subcarriers $(N)$, making the TP proposed estimator suitable for modern OFDM systems like DVB-T2 or for next generation wireless systems that are intended for Intelligent Transportation Systems (ITS) than using the CP-based estimators.

\subsection{CFO Estimation Range}

Systems that can estimate $\varepsilon$ values over a wider range are the more preferable systems to be used. In both Moose and CP schemes the value of estimated $\varepsilon$ does not exceed 0.5. But this estimation range is extended in TS and proposed TP techniques.

\subsection{Computational Complexity}

The computational complexity here is determined by the number of complex multiplications used in estimation process. In Moose, this number is determined by the number of orthogonal subcarriers (N) used in the system. While in $\mathrm{CP}$ technique, this number is limited by the number of uncorrupted samples which is $\left(-\mathrm{N}_{\mathrm{g}}+\mathrm{v}-1\right)$ multiplications in multipath channels with v-taps. This is the same case used in the proposed TP technique, making CP and proposed TP techniques have the lowest computational complexity. The computational complexity increases in TS technique with the increase in the number of repetition patterns (D). In [8], this computational complexity is computed as[N(1-1/D)]. In [14], as it fully exploit all the possible $[\mathrm{D}(\mathrm{D}-1) / 2]$ sets of repetition patterns for auto-correlation to increase its accuracy, each correlation function $A(m)$ needs $\{[N(1-m / D)], m=0: D-1\}$ multiplications at $\beta=\mathrm{D}-1$.

\subsection{Ability to Update the Estimated Value}

As both Moose and TS schemes use a preamble in the beginning of a frame, they assume constant $\varepsilon$ between two frames. So, these techniques cannot update the estimated value if it changed during the frame. Inserting redundancy samples, in every block, for estimation purpose in $\mathrm{CP}$ and proposed TP schemes allows $\hat{\varepsilon}$ to be updated every OFDM symbol block. This permits them to update and compensate for this value once this happens.

\section{Simulations and Results}

This section shows, by simulations using MATLAB, the tradeoffs between Moose, Cyclic Prefix (C.P.), Training Symbol (T.S.) and proposed Training Prefix (T.P.) schemes. These trade-offs consider the reduction of transmitted data rates, the estimated carrier frequency offset ranges that can be covered by the specific scheme and the resultant estimation accuracy. Also the effect of the number of samples that used in estimation process and the ratio between $\left(N_{g} / N\right)$ are considered in this section. The ability of the proposed TP estimator to update the estimated $\mathrm{CFO}$ value during a frame compared to TS preamble scheme is shown also in this section. All simulations are run for signal to noise ratio of $8 \mathrm{~dB}$. 
The effect of $\left(\mathrm{N}_{\mathrm{g}} / \mathrm{N}\right)$ ratio on the normalized transmitted data efficiency per frame is shown in Fig. 4, where $\mathrm{N}=1204$. The frame used in Fig. 4(a) is assumed to have a length of 15 OFDM symbols while Fig. 4(b) assumes higher frame length with 40 OFDM symbols long. Ideally, the whole frame length should contain data symbols but this is not the case because of the added redundancy bits. As shown from the results, in all cases $\mathrm{CP}$ technique has the highest bandwidth efficiency. When $\left(\mathrm{N}_{\mathrm{g}} / \mathrm{N}\right)$ ratio decreases, the more data can be transmitted. Although both Moose and TS use preamble but it was noted from both Figs 4(a) and $4(\mathrm{~b})$ that (for all $\left(\mathrm{N}_{\mathrm{g}} / \mathrm{N}\right)$ values), TS with $\mathrm{D}=4$ has higher bandwidth efficiency than Moose scheme. This is due to the repetition of the whole OFDM block's samples twice in case of Moose.

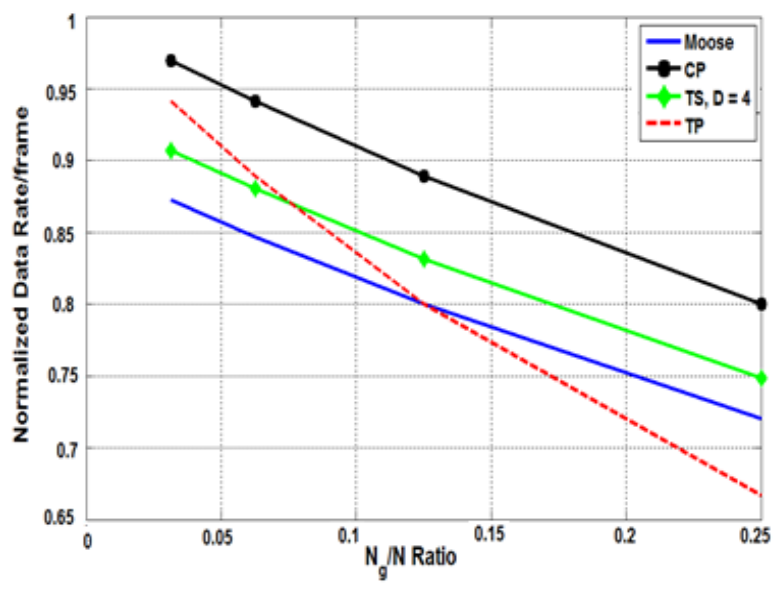

a)

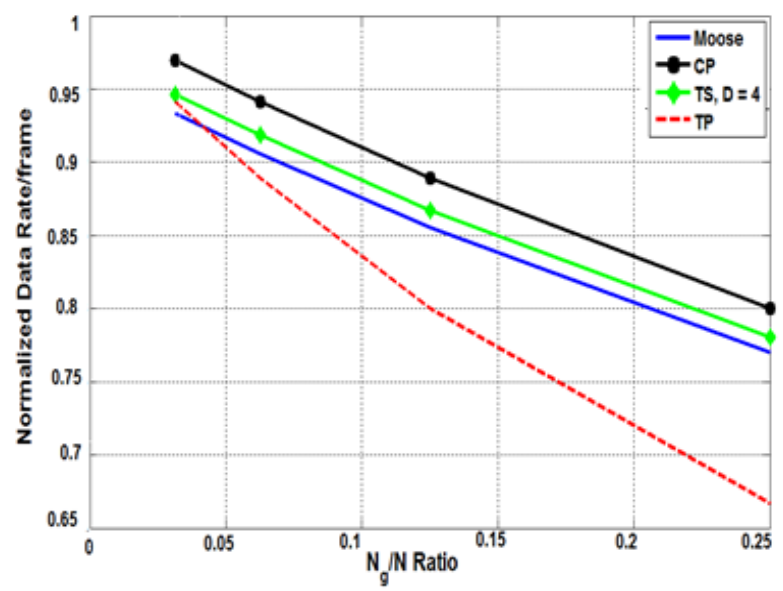

b)

Fig. 4. Data Rate Comparison in Multipath, $\mathbf{N}=1024$, frame length (a) 15 Symbols Blocks, (b) 40 Symbols Blocks

When the frame length increased, as shown in Fig. $4(\mathrm{~b})$, at $\left(\mathrm{N}_{\mathrm{g}} / \mathrm{N}\right)=(1 / 32)$, all techniques has almost the same transmitted bandwidth efficiency. In general, the data rate got at $\left(\mathrm{N}_{\mathrm{g}} / 2 \mathrm{~N}\right)$ in CP-based estimators is equivalent to that at $\left(\mathrm{N}_{\mathrm{g}} / \mathrm{N}\right)$ in case of the TP proposed scheme.

The ability of a specific method to estimate the frequency offset over a wide range is shown in Fig. 5. Simulations in Figs 5(a) and 5(b) are run for $\left(\mathrm{N}_{\mathrm{g}} / \mathrm{N}\right)=(1 / 8)$ and assumed constant offset values during a frame. In both figures, a line with a slope of $45^{\circ}$ and initial value of $(0$, 0 ) indicates the estimation range that can be covered by a specific technique. As shown, both T.S., that uses $\mathrm{D}=8$ and proposed T.P. schemes can cover wider CFO estimation range over Moose and C.P. schemes at the expense of estimation accuracy. This accuracy can be enhanced if the IFFT size increased as shown in Fig. 5(b) which uses $\mathrm{N}=8192$ instead of using $\mathrm{N}=1024$. As a result of using cyclic extension, Moose technique has the lowest estimation range that is lower than 0.5 . 


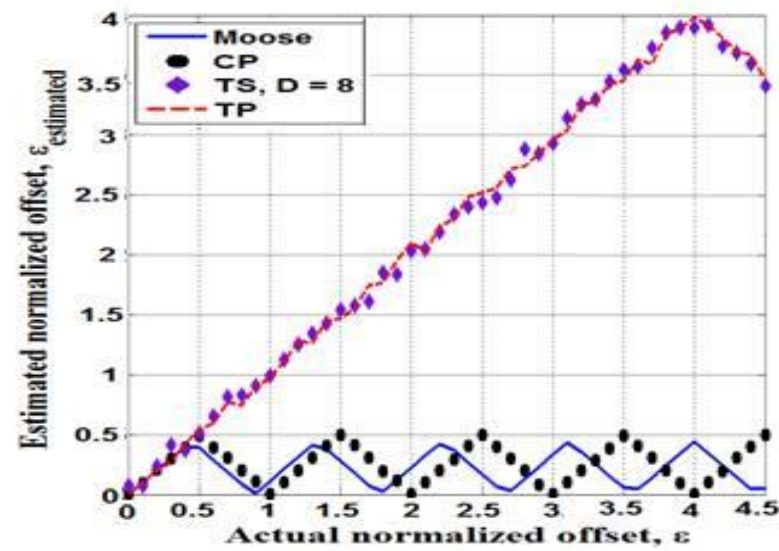

a)

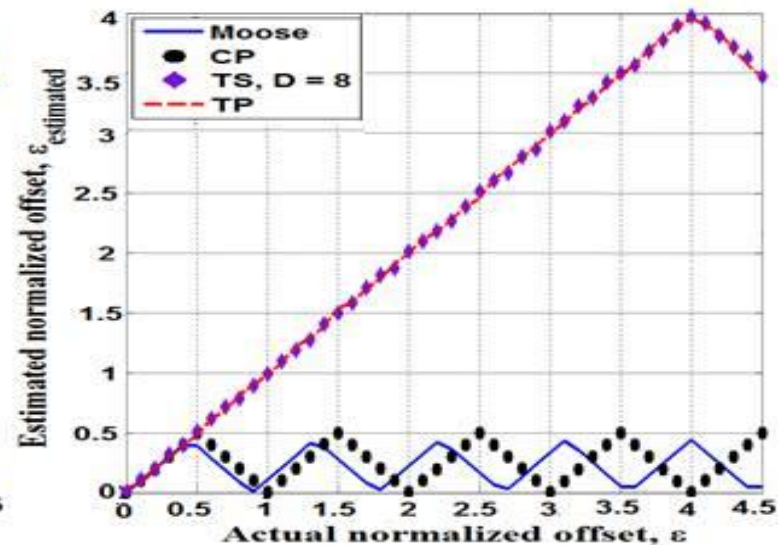

b)

Fig. 5. Estimated $\varepsilon$ versus the actual value in $A W G N, S N R=8 d B$, (a) $N=1024$, (b) $N=8192$

A numerical computational complexity comparison is shown in Fig. 6 at $\left(\mathrm{N}_{\mathrm{g}} / \mathrm{N}\right)=(1 / 8)$ and using one OFDM symbol $(\mathrm{P}=1)$ in estimation process. It was calculated for different number of orthogonal subcarriers $(\mathrm{N}=1 \mathrm{~K}, 2 \mathrm{~K}, 4 \mathrm{~K}, 8 \mathrm{~K}, 16 \mathrm{~K}, 32 \mathrm{~K})$, where $\mathrm{K}=1024$. This computational complexity is measured in this figure in terms of number of multiplications needed in average process. As shown, for all $\mathrm{N}$ values, both $\mathrm{CP}$ and the proposed TP schemes have the lowest computational complexity compared to Moose (that has the highest complexity values) and TS schemes.

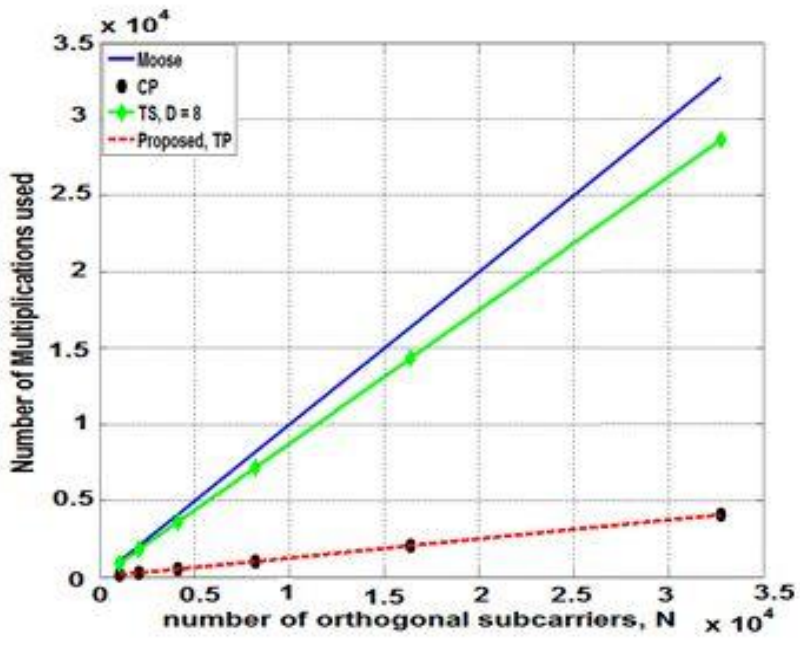

Fig. 7. Estimated $\varepsilon$ versus the actual value in AWGN, N=8192, SNR = 8dB

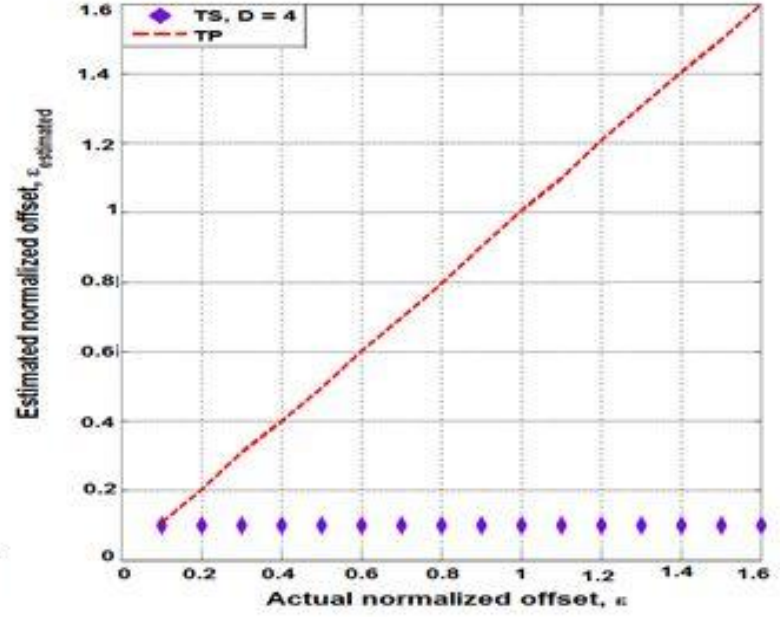

Fig. 6. Computational complexity comparison for different $\mathbf{N}$ values

Assuming constant offset over one OFDM symbol block, the ability of TS and our proposed TP techniques to track the variations during one frame is illustrated in Fig. 7. This figure assumes $\left(\mathrm{N}_{\mathrm{g}} / \mathrm{N}\right)=(1 / 4), 8192$ orthogonal subcarriers and frame consists of 15-OFDM block symbols. As shown, in TS scheme, the initial offset value is estimated in the beginning of the frame and assumed to be constant over the frame length which limits its use in high acceleration conditions. While TP scheme can track those variations in offset values during the frame length which make it preferable to be used in such systems compared to the TS preamble method.

\section{Conclusions}

In this paper, a comparative analysis between different CFO estimation techniques with our TP proposed scheme was performed and verified by simulations. Compared to preamble 
methods, our TP proposed estimator show its ability to update the CFO estimated value many times during the frame while TS preamble method cannot. For large number of orthogonal subcarriers, our proposed TP estimator saves, manifestly, the computational complexity than other schemes with high accuracy. Although these advantages can be obtained for the CPbased blind estimators, the CFO estimation range is maximized in our proposed scheme by four times, at least, compared to this technique. Finally, we can conclude that, for modern OFDM systems that use high number of orthogonal subcarriers, our proposed estimator outperforms all of Moose, CP, and TS schemes at the price of minor reduction of transmitted data rate due to the added redundancy.

\section{References}

1. Shivangi Singh, Santosh Kumar Gupta, Dr. B.S.Rai, "Performance Evaluation to Remove CFO for a Multicarrier Modulation System", IJECT, ISSN : 22307109(Online) | ISSN : 2230-9543(Print), Vol. 2, Issue 3, Sept. 2011

2. Ze Zhu, Xiang Tang, JiZhang Zuo, "Self-Cancellation Method of OFDM ICI", IEEE Wireless Communications, Networking and Mobile Computing, Oct. 2008.

3. Hen-Geul Yeh, Yuan-Kwei Chang, and Babak Hassibi, "A Scheme for Cancelling Intercarrier Interference using Conjugate Transmission in Multicarrier Communication Systems", IEEE transactions on wireless communications, Vol. 6, No. 1, January 2007

4. Giovanni POGGI and Mario TANDA, "Synchronization Techniques for OFDM Systems", PhD in Electrical Engineering and Telecommunications (XVIII cycle), 2005

5. Yaojun Qiao, Zhansheng Wang, and Yuefeng Ji, "Blind frequency offset estimation based on cyclic prefix and virtual subcarriers in CO-OFDM system", Chinese Optics Letters, Vol. 8, No. 9, September 10, 2010

6. Jan-Jaap van de Beek, Magnus Sandell, and Per Ola Borjesson, "ML Estimation of Time and Frequency Offset in OFDM Systems", IEEE Transactions on Signal Processing, Vol. 45, No. 7, July 1997

7. Paul H. Moose, "A Technique for Orthogonal Frequency Division Multiplexing Frequency Offset Correction", IEEE transactions on communications, Vol. 42, No. 10, October 1994

8. Hyoung-Kyu Song, Young-Hwan You, Jong-Ho Paik and Yong-Soo Cho, "Frequency-Offset Synchronization and Channel Estimation for OFDM-Based Transmission", IEEE Communications Letters, Vol. 4, No.3, March 2000

9. Sanjay Sharma, "Digital Signal Processing: with Matlab Programs", fifth edition, July 2009.

10. Santosh Kr. Gupta and Rajan Mishra, "A Comparative Analysis to Remove CFO in OFDM System", VSRD-IJEECE, Vol. 1 (5), 2011

11. Eric W. Weisstein, "CRC Concise Encyclopedia of Mathematics", Second Edition, page 120, ISBN 1-58488-347-2, 2003

12. Yinsheng LIU and Zhenhui TAN, "Carrier Frequency Offset for OFDM Systems using Repetitive Patterns", Radioengineering, Vol. 21, No. 3, September 2012

13. Xiaoli Ma, Georgios B. Giannakis and Sergio Barbarossa, "Non Data Aided Frequency Offset Chanel Estimation in OFDM and Related Block Transmissions", IEEE international conference on communications, Vol. 6, pp. 1866-1870, Jun 2001

14. Shaimaa ElSayed Ibrahim, K.A. ElBarbary and Ragab M. El-Sagheer, "Maximizing CFO Estimation Range Using a New OFDM Symbol Structure", International Journal of Computer Applications, Vol. 88, No.4, February 2014

15. M. Morelli and U. Mengali, "An Improved Frequency Offset Estimator for OFDM Applications", IEEE, Communication Theory Mini-Conference, PP: 106-109, Print ISBN:0-7803-5653-5, Jun. 1999 